\title{
Meta-analysis of Theory of Mind (ToM) impairment in bipolar disorder
}

\author{
E. Bora $^{1 *}$, C. Bartholomeusz ${ }^{1,2}$ and C. Pantelis ${ }^{1}$ \\ ${ }^{1}$ Melbourne Neuropsychiatry Centre, Department of Psychiatry, The University of Melbourne and Melbourne Health, South Carlton, VIC, Australia \\ ${ }^{2}$ Orygen, National Centre of Excellence in Youth Mental Health and The Centre for Youth Mental Health, The University of Melbourne, Parkville, \\ VIC, Australia
}

Background. Theory of mind (ToM) dysfunction is prominent in a number of psychiatric disorders, in particular, autism and schizophrenia, and can play a significant role in poor functioning. There is now emerging evidence suggesting that ToM abilities are also impaired in bipolar disorder (BP); however, the relationship between ToM deficits and mood state is not clear.

Method. We conducted a meta-analysis of ToM studies in BP. Thirty-four studies comparing 1214 patients with BP and 1097 healthy controls were included. BP groups included remitted (18 samples, 545 BP patients), subsyndromal (12 samples, $510 \mathrm{BP}$ patients), and acute (manic and/or depressed) (10 samples, $159 \mathrm{BP}$ patients) patients.

Results. ToM performance was significantly impaired in BP compared to controls. This impairment was evident across different types of ToM tasks (including affective/cognitive and verbal/visual) and was also evident in strictly euthymic patients with BP $(d=0.50)$. There were no significant differences between remitted and subsyndromal samples. However, ToM deficit was significantly more severe during acute episodes $(d=1.23)$. ToM impairment was significantly associated with neurocognitive and particularly with manic symptoms.

Conclusion. Significant but modest sized ToM dysfunction is evident in remitted and subsyndromal BP. Acute episodes are associated with more robust ToM deficits. Exacerbation of ToM deficits may contribute to the more significant interpersonal problems observed in patients with acute or subsyndromal manic symptoms. There is a need for longitudinal studies comparing the developmental trajectory of ToM deficits across the course of the illness.

Received 11 July 2015; Revised 9 September 2015; Accepted 11 September 2015; First published online 12 October 2015

Key words: Bipolar disorder, manic-depressive, neurocognition, theory of mind, social cognition.

\section{Introduction}

Theory of mind (ToM), the ability to infer the mental states of self and others, is one of the most important social cognitive abilities for maintaining effective and adaptive social functioning. ToM dysfunction is a well-established finding in schizophrenia as well as developmental disorders such as autism (Bora et al. 2009a; Senju, 2012; Bora \& Pantelis, 2013; Ventura et al. 2015). ToM impairment can significantly contribute to psychosocial difficulties in psychiatric disorders as social cognitive abilities might be more directly related to interpersonal functioning than general neurocognitive abilities (Fett et al. 2011). In schizophrenia, studies have suggested that neuropsychological deficits and ToM deficits contribute to social dysfunction

* Address for correspondence: Dr E. Bora, Department of Psychiatry, The Melbourne Neuropsychiatry Centre, The University of Melbourne and Melbourne Health, Alan Gilbert Building NNF level 3, Carlton 3053, Australia.

(Email: emrebora@hotmail.com)
(Bora et al. 2006a; Green et al. 2012). A significant proportion of patients with bipolar disorder (BP) also have poor psychosocial functioning (Sanchez-Moreno et al. 2009; Burdick et al. 2010) and neurocognitive impairment is evident in many patients with BP even during remission (Bora et al. 2009b; Burdick et al. 2014; Trotta et al. 2014). It might be expected that ToM deficits might also be associated with BP.

Emerging literature suggests that ToM ability is also impaired in BP (Bora et al. 2005; Samamé et al. 2012). However, there are inconsistent findings regarding the persistence of ToM deficits during euthymia, as both positive and negative findings have been reported. In their meta-analyses of social cognition in BP, Samamé et al. $(2012,2013)$ also conducted a preliminary analysis of 9 and 11 ToM studies, respectively, in 'remitted' BP patients and found significant but modest impairments. However, in these meta-analyses strict euthymia criteria were not used and a number of studies comprised mixed patient samples which included subsyndromal depressive/manic or mild depression (Ioannidi et al. 2013; Lee et al. 2013; Thaler 
et al. 2013). Moreover, it is important to investigate whether ToM deficits are trait- or state-related, as it has been suggested that persistent ToM deficits are risk factors for relapse in mood disorder (Inoue et al. 2006). To date, no meta-analysis has investigated ToM deficits in acute episodes and differences between remitted patients and patients with acute or subsyndromal symptoms.

Another important point to consider is the heterogeneity of ToM as a construct. Mode (i.e. visual $v$. verbal), content [i.e. inferring what a person is feeling (affective) $v$. inferring beliefs and motivations (cognitive)] and complexity (i.e. basic $v$. advanced) of stimuli used in ToM tasks in previous BP studies vary greatly. These aspects of ToM can be related to relatively separate neural networks (Schurz et al. 2014) which can be more or less impaired in BP. For example, some authors have suggested that ToM for cognitive but not emotional stimuli is impaired in BP (Shamay-Tsoory et al. 2009; Montag et al. 2010; Barrera et al. 2013).

In this comprehensive meta-analysis of ToM, we aimed to investigate ToM deficits in BP, including samples with remitted patients, and patients with acute and subsyndromal symptoms, in comparison to healthy controls. This meta-analysis also aimed to explore the effect of clinical, demographic, ToM task type and neurocognitive variables on patient-control differences for ToM.

\section{Method}

\section{Study selection}

We followed MOOSE and PRISMA guidelines in conducting this meta-analysis (Stroup et al. 2000; Moher et al. 2009). A literature search was conducted using the databases Pubmed, PsycINFO, ProQuest and Scopus to identify the relevant studies (January 1990 to June 2015) using the combination of key words as follows: Theory of mind, mentalizing, social cognition, bipolar disorder. The same search was performed in Google Scholar to retrieve unpublished studies. Reference lists of published reports were also reviewed for additional studies. Inclusion criteria were studies that: (1) compared ToM performance of BP patients with that of healthy controls; (2) reported sufficient data to calculate the effect sizes and standard errors of the ToM measures. Studies in pediatric samples and overlapping samples were excluded. We also contacted nine authors for unreported information and clarification (including Dr Ioannidi, Dr Van Rheenen, Dr Purcell, Dr Donohoe, Dr Lahera whose studies are included in the current meta-analysis). A total of 34 studies involving $1214 \mathrm{BP}$ patients (40 samples) and
1097 healthy controls were included in the current meta-analysis (Table 1) (see Supplementary Fig. S1 for flow chart of the study selection process). Percentage of females was very similar in BP (54.7\%) and healthy controls (52.2\%). BP patients were significantly older than healthy controls $[d=0.23,95 \%$ confidence interval (CI) 0.10-0.37, $Z=3.4, p<0.001]$.

We also categorized included studies into three groups: remitted (based on a strict euthymia criteria), subsyndromal (stable non-acute outpatients, including patients with mild manic and depressive symptoms who did not meet criteria for euthymia) and acute (manic and/or depressed). Strict euthymia criteria for the remission group were defined as Hamilton Depression Rating Scale (HAMD) score $<6-10$, Young Mania Rating Scale (YMRS) score $<6-10$ or very small mean HAMD and YMRS scores (mean \pm 2 s.D. within range of euthymia criteria). Eighteen samples including 545 BP patients were classified as remitted based on these criteria. Ten samples included patients (159 $\mathrm{BP}$ ) in acute episode (6 manic, 3 depressive, 1 mixture of manic and depressive). The remaining 12 studies included outpatients (510 BP) with subsyndromal or mild depressive or mild manic symptoms.

\section{ToM measures}

Most commonly used tasks were Reading the Mind in the Eyes Task (RMET; Baron-Cohen et al. 2001) and Faux pas recognition tasks (Stone et al. 1998). Other ToM measures included the Hinting, Happe stories, picture sequencing tasks, The Awareness of Social Inference Test (TASIT)-sarcasm, Movie for Assessment of Social Cognition (MASC) and different versions of false belief and ToM stories and ToM cartoons.

\section{Statistical analyses}

For studies that reported more than one ToM task, pooled effect size and S.E. values were calculated. We also calculated cognitive, affective, verbal and visual ToM scores. Separate task-specific analyses were also conducted if there were at least five studies reporting a particular measure; individual task analyses were possible for the RMET, Hinting task, and the Faux pas task. An additional analysis was conducted for false belief stories (story contents differ).

Meta-analyses were performed using MIX software version 1.7 on a Windows platform (Bax et al. 2006) and in $\mathrm{R}$ environment (OpenMetaAnalyst, Metafor) (Viechtbauer, 2010; Wallace et al. 2012). Effect sizes were weighted using the inverse variance method. A random-effects model (DerSimonian-Laird estimate) was used as the distributions of effect sizes were heterogeneous for the number of variables. 
Table 1. Studies included into the meta-analysis

\begin{tabular}{|c|c|c|c|c|c|c|c|}
\hline Study & Sample & ToM & $\mathrm{BP}$ characteristics & State & $\begin{array}{l}\text { Mood symptoms and } \\
\text { euthymia criteria }\end{array}$ & Cognitive variables & Outcome \\
\hline Andrews $(2013)^{a}$ & $\begin{array}{l}16 \mathrm{BP} \\
20 \mathrm{HC}\end{array}$ & Yoni & $\begin{array}{l}11 \mathrm{BP} \mathrm{I}, 5 \mathrm{BP} \text { II } \\
\text { Age }=41.6 \\
\text { Duration }=21.3\end{array}$ & Remitted & $\begin{array}{c}\mathrm{EC}=\mathrm{HAMD}<6, \mathrm{YMRS}<6, \\
\mathrm{HAMD}=3.3, \mathrm{YMRS}=1.4\end{array}$ & RBANS & BP significantly impaired \\
\hline Barrera et al. (2013) & $\begin{array}{l}12 \mathrm{BP} \\
12 \mathrm{HC}\end{array}$ & Faux pas, RMET & $\begin{array}{l}7 \text { BD II, } 5 \text { BD I } \\
\text { Age }=48.2 \\
\text { Duration }=23.2\end{array}$ & Remitted & $\mathrm{EC}=\mathrm{HAMD}<7, \mathrm{YMRS}<8$ & & $\begin{array}{l}\text { Faux pas impaired in BP } \\
\text { Not related to } \\
\text { functioning }\end{array}$ \\
\hline Bazin et al. (2009) & $\begin{array}{l}15 \mathrm{BP} \\
15 \mathrm{HC}\end{array}$ & Intention & Age $=36.1$ & Manic & YMRS $=19.3$ & & $\begin{array}{l}\text { Impaired compared to } \\
\text { controls }\end{array}$ \\
\hline Bora et al. (2005) & $\begin{array}{l}43 \mathrm{BP} \\
30 \mathrm{HC}\end{array}$ & Hinting, RMET & $\begin{array}{l}\text { All BP I } \\
\text { Age }=38.6 \\
\text { Duration }=15.5 \\
26 / 43 \text { history of psychosis }\end{array}$ & Remitted & $\begin{array}{c}\mathrm{EC}=\mathrm{HAMD}<7, \mathrm{YMRS}<6, \\
\mathrm{HAMD}=1.7, \mathrm{YMRS}=0.7\end{array}$ & $\begin{array}{l}\text { WCST,TMT, CPT, } \\
\text { Stroop, verbal } \\
\text { fluency, verbal } \\
\text { memory }\end{array}$ & $\begin{array}{l}\text { BP impaired in both tasks } \\
\text { Lost significance when } \\
\text { corrected for EF deficit }\end{array}$ \\
\hline Budak $(2011)^{\mathrm{a}}$ & $\begin{array}{l}52 \mathrm{BP} \\
60 \mathrm{HC}\end{array}$ & $\begin{array}{l}\text { RMET, } \\
\text { ToM battery } \\
\text { including FB, } \\
\text { irony, Faux pas, }\end{array}$ & $\begin{array}{l}\text { All BP I } \\
\text { Age }=34.5 \text { Duration }=11.9 \\
47 / 52 \text { history of psychosis }\end{array}$ & Remitted & $\mathrm{EC}=\mathrm{HAMD}<6, \mathrm{YMRS}<6$ & & BP significantly impaired \\
\hline Caletti et al. (2013) & $\begin{array}{l}18 \mathrm{BP} \\
18 \mathrm{HC}\end{array}$ & Faux pas, RMET & $\begin{array}{l}10 \text { BP I, } 8 \text { BP II } \\
\text { Age }=42.2 \\
\text { Duration }=17.5\end{array}$ & Remitted & $\begin{array}{c}\mathrm{EC}=\mathrm{HAMD}<7, \mathrm{YMRS}<10 \\
\mathrm{HAMD}=4.8, \mathrm{YMRS}=2.5\end{array}$ & $\begin{array}{l}\text { Planning, fluency, } \\
\text { Speed, WM, verbal } \\
\text { memory }\end{array}$ & No difference \\
\hline Caponigro (2007) ${ }^{\mathrm{a}}$ & $\begin{array}{l}19 \mathrm{BP} \\
15 \mathrm{HC}\end{array}$ & FB1 and FB2 & Age $=46.5$ & Remitted & $\mathrm{EC}=\mathrm{HAMD}<10, \mathrm{BRMS}<7$ & IQ & $\begin{array}{l}\text { In BP ToM impaired } \\
\text { compared to HC }\end{array}$ \\
\hline Cusi et al. (2012) & $\begin{array}{l}25 \mathrm{BP} \\
25 \mathrm{HC}\end{array}$ & RMET & $\begin{array}{l}17 \text { BP I, } 7 \text { BP II } \\
\text { Age }=45.2 \\
\text { Duration }=23.1\end{array}$ & $\begin{array}{l}\text { Subsyndromal } \\
\text { Mixture of euthymia, } \\
\text { mild depressed }\end{array}$ & $\begin{array}{l}\mathrm{EC}=\mathrm{YMRS}<10, \mathrm{HAMD}= \\
8.1, \mathrm{YMRS}=2.0\end{array}$ & & ToM impaired in BP \\
\hline $\begin{array}{l}\text { Donohoe et al. } \\
\text { (2012) }\end{array}$ & $\begin{array}{l}102 \mathrm{BP} \\
132 \mathrm{HC}\end{array}$ & Hinting, RMET & $\begin{array}{l}\text { Age }=44.8 \\
\text { Duration }=20.6\end{array}$ & Subsyndromal & & $\begin{array}{l}\mathrm{IQ}, \mathrm{WM}, \mathrm{CPT} \text {, verbal } \\
\text { memory }\end{array}$ & ToM impaired in BP \\
\hline Duman $(2014)^{a}$ & $\begin{array}{l}30 \mathrm{BP} \\
30 \mathrm{HC}\end{array}$ & $\begin{array}{l}\text { Hinting, RMET, } \\
\text { Faux pas }\end{array}$ & $\begin{array}{l}\text { All BP I } \\
\text { Age }=36.3 \\
\text { Duration }=9.4\end{array}$ & Remitted & $\begin{array}{c}\mathrm{EC}=\mathrm{HAMD}<8, \mathrm{YMRS}<6 \\
\mathrm{HAMD}=0.3, \mathrm{YMRS}=0.4\end{array}$ & $\begin{array}{l}\text { WCST, TMT, Digit } \\
\text { span, Stroop }\end{array}$ & No difference \\
\hline Ibanez et al. (2012) & $\begin{array}{l}13 \mathrm{BP} \\
13 \mathrm{HC}\end{array}$ & Faux pas, RMET & $\begin{array}{l}\text { All BP II } \\
\text { Age }=40.1\end{array}$ & Remitted & $\mathrm{EC}=\mathrm{BDI}<6, \mathrm{YMRS}<6$ & WM, TMT A and B & Faux pas impaired \\
\hline $\begin{array}{l}\text { Ioannidi et al. } \\
\text { (2013) }\end{array}$ & $\begin{array}{l}55 \mathrm{BP} \\
53 \mathrm{HC}\end{array}$ & $\begin{array}{l}\text { Faux pas, FB1, } \\
\text { Hinting }\end{array}$ & $\begin{array}{l}\text { Age }=41.9 \\
\text { Duration }=14.1\end{array}$ & Subsyndromal & $\mathrm{HAMD}=7.7, \mathrm{YMRS}=5.7$ & & $\begin{array}{l}\text { BP impaired in faux pas } \\
\text { recognition }\end{array}$ \\
\hline
\end{tabular}




\begin{tabular}{|c|c|c|c|c|c|c|c|}
\hline Study & Sample & ToM & $\mathrm{BP}$ characteristics & State & $\begin{array}{l}\text { Mood symptoms and } \\
\text { euthymia criteria }\end{array}$ & Cognitive variables & Outcome \\
\hline $\begin{array}{l}\text { Ioannidi et al. } \\
\text { (2015) }\end{array}$ & $\begin{array}{l}29 \mathrm{BP} \\
29 \mathrm{HC}\end{array}$ & $\begin{array}{l}\text { Faux pas, FB } \\
\text { Hinting }\end{array}$ & $\begin{array}{l}\text { Age }=44.2 \\
\text { Duration }=16.30\end{array}$ & $\begin{array}{l}\text { Acute episode } \\
13 \text { manic } \\
16 \text { depressed }\end{array}$ & & $\begin{array}{l}\text { WCST, TMT, Digit } \\
\text { Span, Stroop, } \\
\text { Verbal memory }\end{array}$ & $\begin{array}{l}\text { BP impaired in all ToM } \\
\text { tasks }\end{array}$ \\
\hline Kerr et al. (2003) & $\begin{array}{l}48 \mathrm{BP} \\
15 \mathrm{HC}\end{array}$ & FB1, FB2 & $\begin{array}{l}\text { Age }=43.9 \\
\text { Duration }=10.9\end{array}$ & $\begin{array}{l}20 \text { manic } \\
15 \text { depressive } \\
13 \text { remitted }\end{array}$ & $\begin{array}{l}\mathrm{BDI}=3.2 / 16.9 / 2.1, \mathrm{BRMS}= \\
13.3 / 2.2 / 2.9\end{array}$ & & $\begin{array}{l}\text { ToM only impaired in } \\
\text { manic and depressed } \\
\text { patients }\end{array}$ \\
\hline Lahera et al. (2008) & $\begin{array}{l}75 \mathrm{BP} \\
48 \mathrm{HC}\end{array}$ & Happe stories & $\begin{array}{l}\text { All BP I } \\
\text { Age }=48.6 \\
42 / 75 \text { history of psychosis }\end{array}$ & Remitted & $\mathrm{EC}=\mathrm{HAMD}<7, \mathrm{YMRS}<7$ & $\begin{array}{l}\text { WCST, sustained } \\
\text { attention }\end{array}$ & ToM impaired in $\mathrm{BP}$ \\
\hline Lahera et al. (2015) & $\begin{array}{l}46 \mathrm{BP} \\
50 \mathrm{HC}\end{array}$ & Hinting & $\begin{array}{l}\text { Age }=38.6 \\
\text { Duration }=15.1\end{array}$ & $\begin{array}{l}\text { Subsyndromal } \\
23 \text { remitted } \\
19 \text { subclinical } \\
4 \text { depressed }\end{array}$ & $\mathrm{EC}=\mathrm{HAMD}<7, \mathrm{YMRS}<7$ & & ToM impaired in BP \\
\hline Lee et al. (2013) & $\begin{array}{l}68 \mathrm{BP} \\
31 \mathrm{HC}\end{array}$ & TASIT 3-sarcasm & $\begin{array}{l}46 \text { BP I, } 22 \text { BP II } \\
\text { Age }=43.9 \\
\text { Duration }=25.6 \\
15 / 46 \text { history of psychosis }\end{array}$ & Subsyndromal & $\mathrm{HAMD}=8.1, \mathrm{YMRS}=3.3$ & $\begin{array}{l}\text { Reasoning, WM, } \\
\text { Speed, Verbal and } \\
\text { Visual memory, } \\
\text { attention }\end{array}$ & $\begin{array}{l}\text { BP not impaired } \\
\text { compared to HC }\end{array}$ \\
\hline $\begin{array}{l}\text { Martino et al. } \\
\text { (2011) }\end{array}$ & $\begin{array}{l}81 \mathrm{BP} \\
34 \mathrm{HC}\end{array}$ & Faux pas, RMET & $\begin{array}{l}45 \text { BP I, } 36 \text { BP II } \\
\text { Age }=39.7 \\
\text { Duration }=11.9\end{array}$ & Remitted & $\mathrm{EC}=\mathrm{HAMD}<8, \mathrm{YMRS}<6$ & $\begin{array}{l}\text { Verbal memory } \\
\text { Digit span, verbal } \\
\text { Fluency, TMT }\end{array}$ & ToM impaired in BP \\
\hline $\begin{array}{l}\text { McKinnon et al. } \\
\text { (2010) }\end{array}$ & $\begin{array}{l}14 \mathrm{BP} \\
14 \mathrm{HC}\end{array}$ & ToM: 1st and 2nd & 8 BP I, 5 BP II, 1 BP NOS & Subsyndromal & $\begin{array}{r}\mathrm{HAMD}=7-15, \mathrm{YMRS}<10 \\
\mathrm{HAMD}=10.8, \mathrm{YMRS}=3.2\end{array}$ & & $\begin{array}{l}\text { BP impaired performance } \\
\text { in } 2 \text { nd order task }\end{array}$ \\
\hline $\begin{array}{l}\text { Montag et al. } \\
\text { (2010) }\end{array}$ & $\begin{array}{l}29 \mathrm{BP} \\
29 \mathrm{HC}\end{array}$ & MASC & $\begin{array}{l}\text { All BP I } \\
\text { Age }=44 \\
\text { Duration }=21\end{array}$ & Subsyndromal & $\begin{array}{l}\mathrm{EC}=\mathrm{HAMD}<14, \mathrm{YMRS} \\
<5, \mathrm{HAMD}=6.7 \mathrm{YMRS}= \\
3.4\end{array}$ & AVLT & ToM impaired in BP \\
\hline Olley et al. (2005) & $\begin{array}{l}15 \mathrm{BP} \\
13 \mathrm{HC}\end{array}$ & $\begin{array}{l}\text { ToM stories, ToM } \\
\text { cartoon }\end{array}$ & $\begin{array}{l}\text { All BP I } \\
\text { Age }=39.2\end{array}$ & Remitted & $\begin{array}{l}\mathrm{EC}=\mathrm{HAMD}<12, \mathrm{YMRS} \\
<12, \mathrm{HAMD}=3.0 \\
\mathrm{YMRS}=1.5\end{array}$ & $\begin{array}{l}\text { SOC,Verbal fluency, } \\
\text { Set shifting, Stroop }\end{array}$ & $\begin{array}{l}\text { Impaired only in verbal } \\
\text { ToM } \\
\text { ToM is significantly } \\
\text { correlated with EF } \\
\text { deficits }\end{array}$ \\
\hline $\begin{array}{l}\text { Ozel-Kizil et al. } \\
(2012)^{\text {a }}\end{array}$ & $\begin{array}{l}18 \mathrm{BP} \\
27 \mathrm{HC}\end{array}$ & Faux pas & $\begin{array}{l}\text { All BP I } \\
\text { History of psychosis }\end{array}$ & Remitted & $\mathrm{EC}=\mathrm{HAMD}<7, \mathrm{YMRS}<7$ & & No significant difference \\
\hline Purcell et al. (2013) & $\begin{array}{l}26 \mathrm{BP} \\
28 \mathrm{HC}\end{array}$ & RMET & $\begin{array}{l}\text { All BP I } \\
\text { Age }=29.6 \\
\text { Duration }=13.4\end{array}$ & Remitted & $\mathrm{EC}=\mathrm{IDS}-\mathrm{C}<11, \mathrm{YMRS}<7$ & LNS & No difference \\
\hline
\end{tabular}




\begin{tabular}{|c|c|c|c|c|c|c|c|}
\hline Robinson $(2010)^{\mathrm{a}}$ & $\begin{array}{l}39 \mathrm{BP} \\
28 \mathrm{C}\end{array}$ & RMET & $\begin{array}{l}\text { Age }=44.9 \\
\text { Duration }=23.3\end{array}$ & Remitted & $\begin{array}{c}\mathrm{EC}=\mathrm{HAMD}<8, \mathrm{YMRS}<8, \\
\mathrm{HAMD}=2.1, \mathrm{YMRS}=1.5\end{array}$ & & No difference \\
\hline $\begin{array}{l}\text { Rossell \& Van } \\
\text { Rheenen (2013) }\end{array}$ & $\begin{array}{l}28 \mathrm{BP} \\
29 \mathrm{HC}\end{array}$ & ToM stories & $\begin{array}{l}\text { All BP I, psychotic } \\
\text { Age }=38.3 \\
\text { Duration }=16.3\end{array}$ & Manic & & & Both group impaired \\
\hline $\begin{array}{l}\text { Rowland et al. } \\
\text { (2013) }\end{array}$ & $\begin{array}{l}33 \mathrm{BP} \\
58 \mathrm{HC}\end{array}$ & $\begin{array}{l}\text { TASIT 2,3- } \\
\text { Sarcasm }\end{array}$ & $\begin{array}{l}\text { All BP I } \\
\text { Age }=40.7\end{array}$ & Subsyndromal & & & BP impaired \\
\hline Sakarya $(2012)^{\mathrm{a}}$ & $\begin{array}{l}30 \mathrm{BP} \\
30 \mathrm{HC}\end{array}$ & $\begin{array}{l}\text { FB1, FB2, } \\
\text { Hinting, Faux } \\
\text { pas }\end{array}$ & $\begin{array}{l}\text { Age }=36.3 \\
\text { Duration }=12.5\end{array}$ & Remitted & $\mathrm{HAMD}=4.2, \mathrm{YMRS}=2.9$ & $\begin{array}{l}\text { IQ, WCST, WM, } \\
\text { Letter fluency }\end{array}$ & BP significantly impaired \\
\hline $\begin{array}{l}\text { Sarfati \& } \\
\text { Hardy-Baylé } \\
(1999)\end{array}$ & $\begin{array}{l}10 \mathrm{BP} \\
15 \mathrm{HC}\end{array}$ & $\begin{array}{l}\text { ToM picSeq } \\
\text { (intention) }\end{array}$ & $\begin{array}{l}\text { All BP I } \\
\text { Age }=33.9 \\
\text { Duration }=6.8\end{array}$ & Manic & & & $\begin{array}{l}\text { BP: No difference } \\
\text { compared to HC }\end{array}$ \\
\hline $\begin{array}{l}\text { Shamay-Tsoory } \\
\text { et al. (2009) }\end{array}$ & $\begin{array}{l}19 \mathrm{BP} \\
20 \mathrm{HC}\end{array}$ & Faux pas, RMET & $\begin{array}{l}\text { All BP I } \\
\text { Age }=40.2 \\
\text { No history of psychosis }\end{array}$ & Remitted & $\mathrm{EC}=\mathrm{HAMD}<9, \mathrm{YMRS}<7$ & İQ, SOC, ID/ED & Faux pas impaired in BP \\
\hline Simon et al. (2013) & $\begin{array}{l}54 \mathrm{BP} \\
34 \mathrm{HC}\end{array}$ & Faux pas & & $\begin{array}{l}26 \text { remitted } \\
28 \text { subsyndromal }\end{array}$ & $\mathrm{HAMD}=7.6, \mathrm{YMRS}=5.9$ & WCST CPT & $\begin{array}{l}\text { Subsyndromal BP } \\
\text { impaired in ToM }\end{array}$ \\
\hline Thaler et al. (2013) & $\begin{array}{l}48 \mathrm{BP} \\
24 \mathrm{HC}\end{array}$ & Hinting, RMET & $\begin{array}{l}\text { All BP I } \\
\text { Age }=35.9 \\
\text { Duration }=19 \\
24 / 48 \text { history of psychosis }\end{array}$ & Subsyndromal & HAMD = 10.1 & IQ & $\begin{array}{l}\text { BP not impaired } \\
\text { compared to HC }\end{array}$ \\
\hline $\begin{array}{l}\text { Van Rheenen \& } \\
\text { Rossell (2013) }\end{array}$ & $\begin{array}{l}49 \mathrm{BP} \\
49 \mathrm{HC}\end{array}$ & $\begin{array}{l}\text { Picture } \\
\text { sequencing }\end{array}$ & $\begin{array}{l}37 \text { BP I, } 12 \text { BP II } \\
\text { Age }=38.5 \\
\text { Duration }=18.1\end{array}$ & Subsyndromal & MADRS $=11.9, \mathrm{YMRS}=6.3$ & & ToM impaired in BP \\
\hline Wiener et al. (2011) & $\begin{array}{l}20 \mathrm{BP} \\
40 \mathrm{HC}\end{array}$ & RMET & $\begin{array}{l}\text { Age }=43 \\
\text { All BP I }\end{array}$ & $\begin{array}{l}\text { Acute episode } \\
8 \text { manic } 12 \text { depressed }\end{array}$ & & & ToM impaired in BP \\
\hline Wolf et al. (2010) & $\begin{array}{l}33 \mathrm{BP} \mathrm{I} \\
29 \mathrm{HC}\end{array}$ & $\begin{array}{l}\text { ToM picSeq and } \\
\text { questions }\end{array}$ & $\begin{array}{l}\text { All BP I } \\
\text { Age }=47.7 \\
\text { Duration }=12.4\end{array}$ & $\begin{array}{l}\text { Subsyndromal Manic } \\
\text { depressed }\end{array}$ & $\begin{array}{l}\mathrm{EC}=\mathrm{HAMD}<15, \\
\mathrm{YMRS}<12\end{array}$ & IQ, WCST Zoo map & ToM impaired in BP \\
\hline
\end{tabular}

BP, Bipolar disorder; HC, healthy controls; EC, euthymia criteria; CPT, Continuous performance test; MASC, Movie for the Assessment of Social Cognition; FB, false belief; SOC, Stockings of Cambridge; ID/ED, intradimensional/extradimensional shift task; EF, executive function; TMT, Trail making task; WM, working memory; WCST, Wisconsin Card Sorting Test; RMET, Reading the Mind in the Eyes Task RBANS, Repeatable Battery for the Assessment of Neuropsychological Status; YMRS, Young Mania Rating Scale; HAMD, Hamilton Depression Rating Scale; BRMS, Bech-Rafaelsen Mania Scale; MADRS, Montgomery-Åsberg Depression Rating Scale; IDS-C, Inventory for Depressive Symptomatology - Clinician; BDI, Beck Depression Inventory.

${ }^{\text {a }}$ Unpublished thesis or conference paper. 
Homogeneity of the distribution of weighted effect sizes was tested with $I^{2}$ and $Q$ tests. Tau squared $\left(\tau^{2}\right)$, an estimate of between-study variance was used as a measure of heterogeneity in the random-effects model. Publication bias was assessed by Egger's test and the Fail-safe $N$ test. Egger's test relies on the theory that studies involving small sample sizes would be more likely to be reported for significant rather than negative findings, while large-scale studies would be more likely to be published regardless of significance of the findings. Fail-safe $N$ test involves computing a combined $p$ value for all studies included in the meta-analysis, and calculating how many additional studies with a zero effect (average $z$ of zero) would be necessary to create a non-significant $p$ (Rosenthal \& Rosnow, 1991).

Meta-regression analyses were conducted for age, gender, duration of education, duration of illness, age of onset, YMRS, HAMD and cognitive impairment in BP-control comparisons whenever at least 10 studies reported these variables. As a measure of cognitive impairment, we used global cognition [current IQ or mean effect sizes calculated as the average of effect sizes of available cognitive domains (verbal, visual memory, working memory, attention, reasoning and problem solving, processing speed); Nuechterlein et al. 2004]. Premorbid IQ and control measures for ToM were not included. Another potential important moderator is the effect of history of psychosis on ToM impairment. Four of the studies have reported separate data to calculate effect sizes for the ToM differences between BP patients with and without history of psychosis. We conducted a preliminary analysis to explore the effect of history of psychosis on ToM based on these studies (Bora et al. 2005; Lahera et al. 2008; Lee et al. 2013; Thaler et al. 2013). Meta-regression analyses (weighted generalized least squares regressions) were conducted using SPSS software (SPSS Inc., USA). Meta-regression analyses performed with a random-effects model were conducted using the restricted-information maximum likelihood method with a significance level set at $p<0.05$. Subgroup analyses were conducted for patient group [remitted, subsyndromal, acute (and manic only)], subtype (BP I), peer review status (journal articles $v$. theses/conference papers). The $Q_{\text {bet }}$ test was used to compare effect sizes of subgroups.

\section{Results}

\section{ToM in BP}

ToM performance of patients with BP was significantly impaired compared to controls (Cohen's $d=0.63$ ) (Fig. 1). ToM impairment in BP I patients in comparison to healthy controls were very similar to the primary analysis ( $d=0.68,95 \%$ CI $0.50-0.87, Z=7.3, p<0.001$ ).
Distribution of effect sizes was heterogeneous $\left(I^{2}=\right.$ $36.5 \%, p=0.02)$ but the magnitude of this heterogeneity was very small in the random-effects model $\left(\tau^{2}=0.04\right)$. There was some evidence of publication bias but the failsafe number was very high $(n=1712)$, suggesting that ToM impairment in BP is a robust effect (Table 2 ).

In 17 studies that assessed general cognition, there was a significant impairment in $\mathrm{BP}(d=0.57,95 \%$ CI $0.45-0.69$, $Z=9.4, p<0.001)$. The severity of ToM impairment in this subset of studies was similar $(d=0.55,95 \%$ CI $0.42-0.68$, $Z=8, p<0.001)$ to general cognitive impairment, as well as ToM deficit in the main analysis $(d=0.63)$. There was no significant difference for ToM impairment between seven studies that were not peer-reviewed and others $\left(d=0.62 v .0 .63, Q_{\text {bet }}=0.20, p>0.05\right)$.

\section{ToM and mood state}

ToM impairment was also evident in euthymic patients in comparison to controls $(d=0.50)$ (Table 2). Distribution of the effect sizes in euthymic patients was homogeneous in the random-effects model. ToM deficit in subsyndromal BP was also significant $(d=0.72)$ and there were no significant differences between remitted and subsyndromal patients $\left(Q_{\text {bet }}=0.97, p=0.32\right)$. By contrast, ToM deficit was much more robust in acute BP patients $(d=1.23)$ and ToM impairment in these patients were significant in comparison to remitted $\left(Q_{\text {bet }}=30.9, \quad p<0.001\right)$ and subsyndromal $\left(Q_{\text {bet }}=23.7\right.$, $p<0.001) \mathrm{BP}$ patients. When analysis for acute BP patients were repeated only for manic patients, the effect size for ToM impairment in manic patients was large $(d=1.31)$ (Table 2$)$.

\section{ToM and task type}

Verbal v. visual

ToM impairments in BP patients compared to controls on verbal $\left(d=0.58,95 \%\right.$ CI $0.48-0.69, Z=10.7, p<0.001, \tau^{2}=$ 0 ) and visual $(d=0.58,95 \%$ CI $0.41-0.74, Z=7.0, p<0.001$, $\left.\tau^{2}=0.08\right)$ tasks were very similar.

\section{Cognitive v. affective}

Both cognitive ToM $(d=0.68,95 \%$ CI $0.56-0.79, Z=11.4$, $\left.p<0.001, \tau^{2}=0.03\right)$ and affective ToM $(d=0.46,95 \% \mathrm{CI}$ $0.28-0.65, Z=4.9, p<0.001, \tau^{2}=0.07$ ) were significantly impaired in BP. In the 12 studies that assessed both cognitive $(d=0.65)$ and affective $(d=0.46)$ ToM, between-group differences were not significant $\left(Q_{\text {bet }}=1.17, p>0.05\right)$.

\section{Individual task analyses}

In individual task analyses, there were significant impairments in the Faux pas task $(d=0.57)$, The Hinting task $(d=0.47)$, the false beliefs tasks $(d=0.53)$ 
Studies

\begin{tabular}{|c|c|c|c|}
\hline Simon et al. 2013 & 0.850 & (0.399, & $1.301)$ \\
\hline Kerr et al. 2003 & 0.740 & $(0.132$, & 1.348) \\
\hline Wiener et al. 2011 & 1.350 & $(0.782$, & $1.918)$ \\
\hline Robinson 2010 & 0.030 & $(-0.460$, & $0.520)$ \\
\hline Barrera et al. 2012 & 0.690 & $(-0.153$, & $1.533)$ \\
\hline Ozel kizil et al. 2012 & 0.610 & $(-0.017$, & $1.237)$ \\
\hline Budak 2011 & 1.130 & $(0.738$ & $1.522)$ \\
\hline Lahera et al. 2015 & 0.480 & $(0.068$, & $0.892)$ \\
\hline Van Rheenen\&Rossell 2013 & 0.550 & $(0.138$, & $0.962)$ \\
\hline Mckinnon 2010 & 0.890 & $(0.106$, & $1.674)$ \\
\hline Cusi et al. 2012 & 0.680 & $(0.112$, & $1.248)$ \\
\hline loannedi et al. 2013 & 0.330 & $(-0.042$, & $0.702)$ \\
\hline Rowland et al. 2013 & 0.330 & $(-0.101$, & $0.761)$ \\
\hline Montag et al. 2009 & 0.840 & (0.291, & 1.389) \\
\hline Bazin et al. 2009 & 1.410 & $(0.626$, & $2.194)$ \\
\hline Rossell \&Van Rheenen 2013 & 0.920 & $(0.371$, & $1.469)$ \\
\hline Sarfati et al. 1999 & 1.030 & $(0.187$, & 1.873) \\
\hline Donohoe et al. 2012 & 0.460 & $(0.186$, & $0.734)$ \\
\hline Lahera et al. 2008 & 0.560 & $(0.188$, & $0.932)$ \\
\hline Martino et al. 2011 & 0.370 & $(-0.042$, & $0.782)$ \\
\hline Lee et al. 2013 & 0.410 & $(-0.021$, & $0.841)$ \\
\hline Bora et al. 2005 & 0.670 & $(0.180$, & $1.160)$ \\
\hline Thaler et al. 2013 & 0.330 & $(-0.160$ & $0.820)$ \\
\hline Sakarya 2012 & 0.640 & (0.111, & $1.169)$ \\
\hline Duman 2014 & 0.470 & $(-0.059$, & $0.999)$ \\
\hline Wolf et al. 2010 & 1.350 & $(0.801$ & $1.899)$ \\
\hline loannedi et al. 2015 & 0.900 & (0.351, & $1.449)$ \\
\hline Purcell et al. 2013 & 0.010 & $(-0.519$, & $0.539)$ \\
\hline Shamay Tsoory et al. 2009 & 0.630 & $(-0.017$, & $1.277)$ \\
\hline Caletti et al. 2013 & 0.220 & $(-0.446$, & $0.886)$ \\
\hline Andrews 2013 & 0.660 & $(-0.026$ & $1.346)$ \\
\hline Caponigro 2007 & 0.780 & $(0.074$, & 1.486) \\
\hline Olley et al. 2005 & 0.570 & $(-0.194$, & 1.334) \\
\hline Ibanez et al. 2012 & 1.000 & $(0.177$, & $1.823)$ \\
\hline Overall $\left(\mathbf{l}^{2}\right.$ & 0.631 & $(0.519$, & $0.744)$ \\
\hline
\end{tabular}

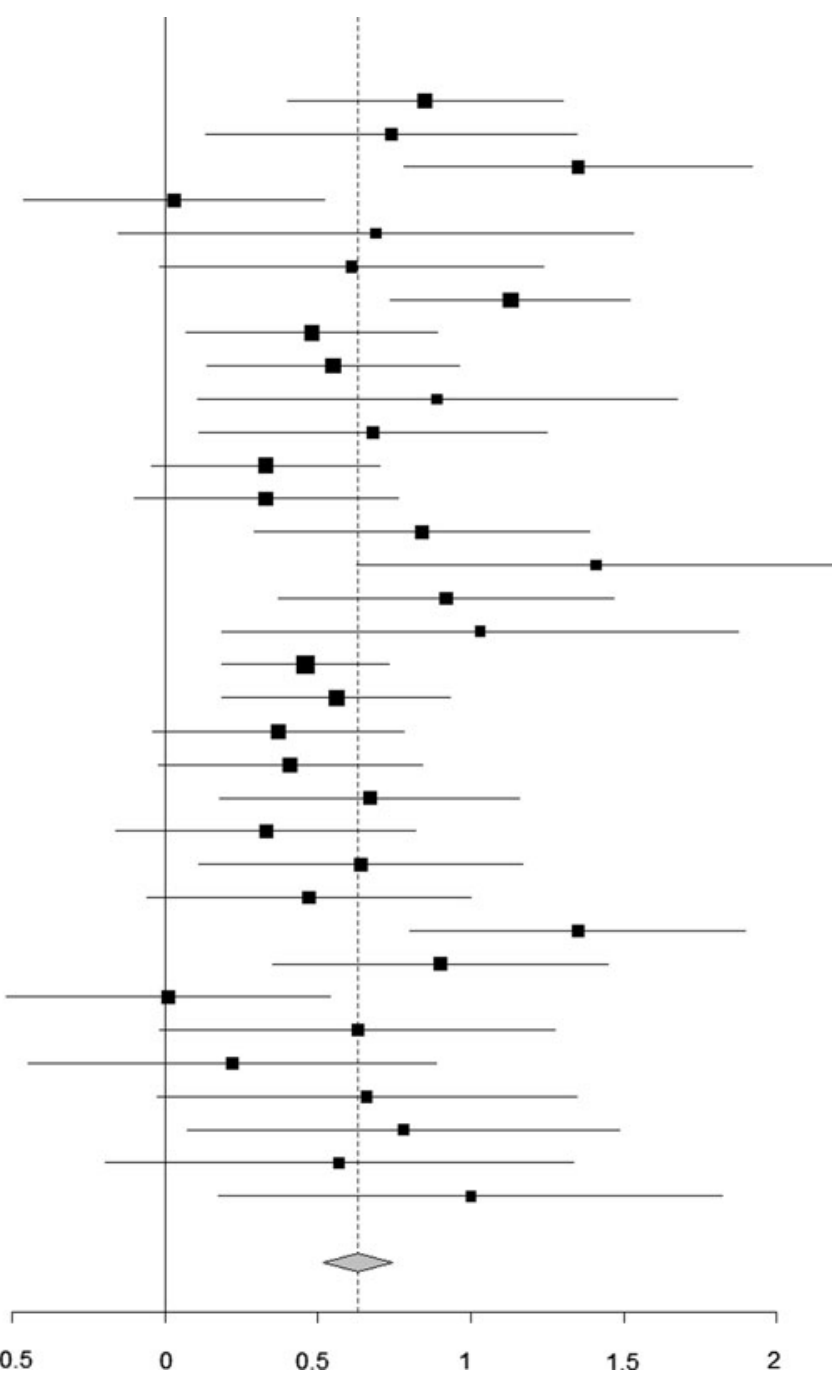

Fig. 1. Forest plot for ToM differences between bipolar disorder and healthy controls.

and the RMET $(d=0.50)$ in comparison to healthy controls (Table 2). There was significant heterogeneity in the distribution of the effect sizes only in the RMET. It was also possible to conduct individual task analyses for two ToM measures in remitted BP patients: Both RMET $(d=0.40)$ and Faux pas recognition $(d=0.50)$ performances were impaired in BP in comparison to controls. There was evidence for publication bias for the Faux pas task, but the fail-safe number was very high $(n=116)$ suggesting that Faux pas recognition impairment in BP was a real effect.

\section{Effect of history of psychosis on ToM impairment}

There was a non-significant trend-level effect of history of psychosis on ToM performance in BP. BP patients with history of psychosis tended to perform poorer than BP without history of psychosis $(d=0.25,95 \%$ CI -0.03 to $0.53, p=0.08$ ).

\section{Meta-regression analyses}

Higher YMRS scores were significantly related to the severity of ToM deficit $(Z=2.90, p=0.004)$. The relationship between HAMD and ToM impairment was less pronounced and was not statistically significant $(Z=1.59, p=0.11)$. Global cognitive impairment was significantly associated with ToM impairment in BP $(Z=4.19, p<0.0001)$. However, to explore possible effects of clinical and demographical variables on ToM which can be masked by acute symptoms, we conducted the following analyses in non-acute patients only (remitted and subsyndromal). There was again a significant effect of global cognitive impairment $(Z=2.90, p=0.004)$ on ToM deficit. There was no significant effect of gender $(Z=0.15, p=0.89)$, age $(Z=0.62$, $p=0.63)$, education $(Z=0.47, p=0.64)$, illness duration $(Z=0.30, p=0.77)$ and age of onset of illness $(Z=1.08$, $p=0.28)$ on ToM in BP. 
Table 2. Mean weighted effect sizes for ToM differences between patients with bipolar disorder and healthy controls

\begin{tabular}{|c|c|c|c|c|c|c|c|c|c|c|c|}
\hline Test BP-HC & $N$ & $\mathrm{BP}$ & $\mathrm{HC}$ & $d$ & $95 \% \mathrm{CI}$ & $Z$ & $p$ & $Q$ test $(p)$ & $\tau^{2}$ & Bias $(p)$ & Fail-safe $N$ \\
\hline \multicolumn{12}{|l|}{ Full sample } \\
\hline ToM & 34 & 1214 & 1097 & 0.63 & $0.52-0.74$ & 11.0 & $<0.001$ & 0.02 & 0.04 & 0.04 & 1712 \\
\hline RMET & 14 & 528 & 514 & 0.50 & $0.29-0.71$ & 4.7 & $<0.001$ & $<0.01$ & 0.08 & 0.69 & 181 \\
\hline Faux pas & 11 & 383 & 330 & 0.57 & $0.40-0.73$ & 6.8 & $<0.001$ & 0.43 & 0 & 0.02 & 137 \\
\hline Hinting & 7 & 379 & 329 & 0.47 & $0.28-0.66$ & 4.9 & $<0.001$ & 0.22 & 0.02 & 0.14 & 59 \\
\hline FB stories & 6 & 220 & 185 & 0.53 & $0.30-0.76$ & 4.5 & $<0.001$ & 0.32 & 0.01 & 0.76 & 32 \\
\hline \multicolumn{12}{|l|}{ Remitted } \\
\hline ToM & 18 & 545 & 475 & 0.50 & $0.35-0.66$ & 6.0 & $<0.001$ & 0.08 & 0.04 & 0.80 & 242 \\
\hline RMET & 10 & 337 & 273 & 0.40 & $0.15-0.65$ & 3.2 & 0.004 & 0.03 & 0.08 & 0.67 & 47 \\
\hline Faux pas & 9 & 269 & 248 & 0.50 & $0.28-0.72$ & 4.4 & $<0.001$ & 0.21 & 0.03 & 0.08 & 60 \\
\hline \multicolumn{12}{|l|}{ Acute } \\
\hline ToM & 10 & 159 & 172 & 1.23 & $1.01-1.45$ & 11.0 & $<0.001$ & 0.62 & 0 & 0.05 & 312 \\
\hline ToM (manic) & 6 & 91 & 143 & 1.31 & $1.0-1.62$ & 8.3 & $<0.001$ & 0.37 & 0.01 & 0.21 & 115 \\
\hline \multicolumn{12}{|l|}{ Subsyndromal } \\
\hline ToM & 12 & 510 & 528 & 0.72 & $0.45-1.0$ & 5.1 & $<0.001$ & $<0.01$ & 0.17 & 0.03 & 300 \\
\hline
\end{tabular}

$\mathrm{CI}$, Confidence interval; BP, bipolar disorder; HC, healthy controls; $d$, Cohen's $d$; RMET, Reading the Mind in the Eyes Task; FB, false belief.

\section{Discussion}

The current meta-analysis investigated ToM performance in a large sample of BP patients in comparison with healthy controls. Our findings showed that ToM ability was significantly impaired in BP in comparison to healthy participants. ToM dysfunction was significantly more pronounced in acute episodes but was also evident in remitted patients.

The effect size of ToM dysfunction $(d=0.63)$ in BP suggests a medium-sized impairment. ToM dysfunction in euthymic BP patients $(d=0.50)$ was comparable to most other neuropsychological deficits observed in the literature (Bora et al. 2009b). Acute phases of BP, especially manic episodes, was associated with exacerbation of ToM deficits and YMRS scores were significantly related to the severity of ToM impairment. Differences between manic and remitted patients were relatively large compared to most traditional neuropsychological domains in which evidence suggests only subtle effects of mood state on cognition (Kurtz \& Gerraty, 2009). However, similar to ToM performance, decision making, impulsivity, insight and some aspects of neurocognition (i.e. commission errors in the continuous performance test) are significantly more impaired during mania in comparison to remission (Bora et al. 2006b; Clark \& Sahakian, 2008). Mania-related functional changes in the ventral prefrontal cortex which has a critical role in reward, decision making and ToM, could potentially explain these findings (Rubinsztein et al. 2001). It could be argued that ToM dysfunction during manic episodes may be related to thought disorder, psychosis, and/or impairment in functioning. However, more studies are needed to be conclusive on this topic.

It is evident that ToM deficits in BP persist in remission. Thus, it could be argued that ToM deficits might be phenotypic risk markers of BP and may contribute to the social dysfunction that is observed in many people with BP during remission. There is sufficient evidence supporting such a link between social impairment and ToM deficits in schizophrenia (Bora et al. 2006a; Green et al. 2012). However, very few studies have examined the link between ToM dysfunction and social functioning in BP (Lahera et al. 2012; Caletti et al. 2013; Lee et al. 2013; Purcell et al. 2013). There is evidence suggesting that social cognitive training programs might be beneficial to improve these abilities in schizophrenia (Kurtz \& Richardson, 2012). The inclusion of social cognition training in cognitive remediation strategies in BP can potentially improve social functioning in this disorder.

Magnitudes of both social and non-social cognitive impairment in BP were similar and relatively modest compared to similarly larger effect sizes for both domains observed in meta-analyses of schizophrenia studies (Dickinson et al. 2007; Bora et al. 2009a). This finding does not support the notion of BP is associated more severe impairment in non-social than social cognition while schizophrenia is associated with the opposite pattern (Lee et al. 2013). However, another important subject is the origin of ToM deficits in BP. It could be argued that ToM impairments in BP are just epiphenomena of neurocognitive impairment, or 
at least there might be partial overlap in deficits in neurocognition and social cognition (Bora et al. 2009c). Our findings suggest that neurocognitive deficits may significantly contribute to ToM deficits in BP given that meta-regression analyses found a significant relationship between ToM and general cognitive impairment. A number of previous studies also proposed that ToM deficits in BP might be secondary to cognitive deficits (Caletti et al. 2013; Lee et al. 2013; Thaler et al. 2013; Bora et al. 2005). For example, a previous study in euthymic BP also supports this notion, as the significant ToM dysfunction in this patient population was no longer significant following correction for working memory deficits (Bora et al. 2005). ToM impairment observed in schizophrenia can also be partly explained by neurocognitive deficits (Sergi et al. 2007; Bora et al. $2009 a-c)$. However, in schizophrenia, it has been shown that neurocognition and social cognition are partly overlapping but relatively unique constructs. In $\mathrm{BP}$, there is a need for further studies investigating the separability of neurocognition and social cognition.

Some authors suggest that BP is only associated with impairment in 'cognitive' but not in 'affective' ToM (Shamay-Tsoory et al. 2009; Montag et al. 2010). However, findings from the current meta-analysis do not support this suggestion, as BP was associated with deficits in both types of tasks. While the effect size for impairment in 'cognitive' ToM was moderately higher than 'affective' ToM, the difference between these two task types was not statistically significant. Contradictory findings might be related to low power of past studies. Donohoe et al. (2012), who had a considerably larger sample size than other past studies, was able to demonstrate a similar degree of impairment in both affective and cognitive ToM. Moreover, differences in some studies can reflect differences in task difficulties rather than task type. Another consideration for future research is the potential effect of history of psychosis on ToM deficits in BP. History of psychosis in BP has been associated with more severe cognitive deficits, especially in some executive abilities (planning and working memory) (Bora et al. 2010). Our findings suggested a similar trend for ToM in BP $(d=0.25)$. However, number of studies were small and between-group did not reach significance $(p=0.08)$. There is a need for further studies to investigate the relationship between ToM impairment and psychosis in BP.

There are some limitations regarding the current meta-analysis. First, it is important to note that a number of different ToM tasks have been used in these studies and task difficulty can certainly play a role in between-group differences. Many studies have not included a neurocognitive assessment battery and/or a control condition/task for the ToM measure. We were only able to examine the effect of general cognition but not executive functions, working memory and other cognitive domains on ToM impairment. Another issue is the development of observed ToM deficits. We did not find evidence for a relationship between more severe ToM deficits and longer duration of illness, or the younger age of illness onset. However, it is premature to conclude neurodevelopmental and/or possible progressive aspects of ToM deficits in BP due to a lack of data in first-episode, at risk samples and early-onset samples as well as longitudinal research. Another consideration for future research is the potential effect of history of psychosis on ToM deficits in BP. History of psychosis in BP has been associated with more severe cognitive deficits, especially in some executive abilities (planning and working memory) (Bora et al. 2010). However, we were not able to investigate the relationship between ToM impairment and psychosis in BP as only a few studies investigated the relationship between these variables.

In conclusion, ToM abilities are impaired in BP. ToM dysfunction is more pronounced during acute episodes, but deficits are also present in remission, suggesting that ToM impairment might be a trait-marker of BP. There is a need for first-episode studies and longitudinal studies comparing the developmental course of ToM and other social cognition abilities in individuals at risk for BP.

\section{Supplementary material}

For supplementary material accompanying this paper visit http://dx.doi.org/10.1017/S0033291715001993.

\section{Acknowledgements}

Prof Christos Pantelis was supported by a NHMRC Senior Principal Research Fellowship (ID 628386).

\section{Declaration of Interest}

Over the last 2 years, Christos Pantelis has participated on Advisory Boards for Janssen-Cilag and Lundbeck. He has received honoraria for talks presented at educational meetings organized by AstraZeneca, Shire, Janssen-Cilag and Lundbeck. The remaining authors have no conflict of interests to report.

\section{References}

Andrews SC (2013). Exploring Social Cognition and Mirror Systems in Schizophrenia and Bipolar Disorder. Monash University. (http://arrow.monash.edu.au/hdl/1959.1/ 909606). 
Barrera A, Vázquez G, Tannenhaus L, Lolich M, Herbst L (2013). Theory of mind and functionality in bipolar patients with symptomatic remission. Revista Psiquiatria y Salud Mental 6, 67-74.

Baron-Cohen S, Wheelwright S, Hill J, Raste Y, Plumb I (2001). The 'Reading the Mind in the Eyes' Test revised version: a study with normal adults, and adults with Asperger syndrome or high-functioning autism. Journal of Child Psychology and Psychiatry 42, 241-251.

Bax L, Yu LM, Ikeda N, Tsuruta H, Moons KGM (2006). Development and validation of MIX: comprehensive free software for meta-analysis of causal research data. BMC Medical Research Methodology 6, 50.

Bazin N, Brunet-Gouet E, Bourdet C, Kayser N, Falissard B, Hardy-Baylé MC, Passerieux C (2009). Quantitative assessment of attribution of intentions to others in schizophrenia using an ecological video-based task: a comparison with manic and depressed patients. Psychiatry Research 167, 28-35.

Bora E, Eryavuz A, Kayahan B, Sungu G, Veznedaroglu B (2006a). Social functioning, theory of mind and neurocognition in outpatients with schizophrenia: mental state decoding may be a better predictor of social functioning than mental state reasoning. Psychiatry Research 145, 95-103.

Bora E, Pantelis C (2013). Theory of mind impairments in first-episode psychosis, individuals at ultra-high risk for psychosis and in first-degree relatives of schizophrenia: systematic review and meta-analysis. Schizophrenia Research 144, 31-36.

Bora E, Vahip S, Akdeniz F (2006b). Sustained attention deficits in manic and euthymic patients with bipolar disorder. Progress in Neuropsychopharmacology and Biological Psychiatry 30, 1097-1102.

Bora E, Vahip S, Gonul AS, Akdeniz F, Alkan M, Ogut M, Eryavuz A (2005). Evidence for theory of mind deficits in euthymic patients with bipolar disorder. Acta Psychiatrica Scandinavica 112, 110-116.

Bora E, Yucel M, Pantelis C (2009a). Theory of mind impairment in schizophrenia: meta-analysis. Schizophrenia Research 109, 1-9.

Bora E, Yucel M, Pantelis C (2009b). Cognitive endophenotypes of bipolar disorder: a meta-analysis of neuropsychological deficits in euthymic patients and their first-degree relatives. Journal of Affective Disorders 113, $1-20$.

Bora E, Yücel M, Pantelis C (2009c). Theory of mind impairment: a distinct trait-marker for schizophrenia spectrum disorders and bipolar disorder? Acta Psychiatrica Scandinavica 120, 253-264.

Bora E, Yücel M, Pantelis C (2010). Neurocognitive markers of psychosis in bipolar disorder: a meta-analytic study. Journal of Affective Disorders 127, 1-9.

Budak EA (2011). Theory of mind and its relationship with clinical features and social functioning in remitted bipolar patients. Thesis. Istanbul. Bakırkoy Research and Training Hospital.

Burdick KE, Goldberg JF, Harrow M (2010). Neurocognitive dysfunction and psychosocial outcome in patients with bipolar I disorder at 15-year follow-up. Acta Psychiatrica Scandinavica 122, 499-506.

Burdick KE, Russo M, Frangou S, Mahon K, Braga RJ, Shanahan M, Malhotra AK (2014). Empirical evidence for discrete neurocognitive subgroups in bipolar disorder: clinical implications. Psychological Medicine 44, 3083-3096.

Caletti E, Paoli RA, Fiorentini A, Cigliobianco M, Zugno E, Serati M, Orsenigo G, Grillo P, Zago S, Caldiroli A, Prunas C, Giusti F, Consonni D, Altamura AC (2013). Neuropsychology, social cognition and global functioning among bipolar, schizophrenic patients and healthy controls: preliminary data. Frontiers in Human Neuroscience 7, 661.

Caponigro JM (2007). Social Cognitive Deficits in Schizophrenia, Schizoaffective Disorder, and Bipolar Disorder: Similarities and Differences. University of Pittsburgh: Pittsburgh.

Clark L, Sahakian BJ (2008). Cognitive neuroscience and brain imaging in bipolar disorder. Dialogues in Clinical Neuroscience 10, 153-163.

Cusi AM, Macqueen GM, McKinnon MC (2012). Patients with bipolar disorder show impaired performance on complex tests of social cognition. Psychiatry Research 200, 258-264.

Dickinson D, Ramsey ME, Gold JM (2007). Overlooking the obvious: a meta-analytic comparison of digit symbol coding tasks and other cognitive measures in schizophrenia. Archives of General Psychiatry 64, 532-542.

Donohoe G, Duignan A, Hargreaves A, Morris DW, Rose E, Robertson D, Cummings E, Moore S, Gill M, Corvin A (2012). Social cognition in bipolar disorder versus schizophrenia: comparability in mental state decoding deficits. Bipolar Disorders 14, 743-748.

Duman T (2014). Theory of mind and executive functions in bipolar disorder patients and their first-degree relatives. Pamukkale University (Thesis).

Fett AK, Viechtbauer W, Dominguez MD, Penn DL, van Os J, Krabbendam L (2011). The relationship between neurocognition and social cognition with functional outcomes in schizophrenia: a meta-analysis. Neuroscience and Biobehavioral Reviews 35, 573-588.

Green MF, Bearden CE, Cannon TD, Fiske AP, Hellemann GS, Horan WP, Kee K, Kern RS, Lee J, Sergi MJ, Subotnik KL, Sugar CA, Ventura J, Yee CM, Nuechterlein KH (2012). Social cognition in schizophrenia, part 1 : performance across phase of illness. Schizophrenia Bulletin 38, 854-864.

Ibanez A, Urquina H, Petroni A, Baez S, Lopez V, do Nascimento M, Herrera E, Guex R, Hurtado E, Blenkmann A, Beltrachini L, Gelormini C, Sigman $M$, Lischinsky A, Torralva T, Torrente F, Cetkovich M, Manes F (2012). Neural processing of emotional facial and semantic expressions in euthymic bipolar disorder (BD) and its association with theory of mind (ToM). PLoS ONE 7, e46877.

Inoue Y, Yamada K, Kanba S (2006). Deficit in theory of mind is a risk for relapse of major depression. Journal of Affective Disorders 95, 125-127.

Ioannidi N, Konstantakopoulos G, Ploumpidis D, Typaldou M, Sakkas D, Papadimitriou GN, Oulis P (2013). Empathy 
and theory of mind in euthymic bipolar disorder. European Psychiatry 28 (Suppl. 1), 1262.

Ioannidi N, Konstantakopoulos G, Sakkas D, Oulis P (2015). The relationship of Theory of Mind with symptoms and cognitive impairment in bipolar disorder: a prospective study. Psychiatriki 26, 17-27.

Kerr N, Dunbar RI, Bentall RP (2003). Theory of mind deficits in bipolar affective disorder. Journal of Affective Disorders 73, 253-259.

Kurtz MM, Gerraty RT (2009). A meta-analytic investigation of neurocognitive deficits in bipolar illness: profile and effects of clinical state. Neuropsychology 23, 551-562.

Kurtz MM, Richardson CL (2012). Social cognitive training for schizophrenia: a meta-analytic investigation of controlled research. Schizophrenia Bulletin 38, 1092-1104.

Lahera G, Herrera S, Reinares M, Benito A, Rullas M, González-Cases J, Vieta E (2015). Hostile attributions in bipolar disorder and schizophrenia contribute to poor social functioning. Acta Psychiatrica Scandinavica 131, 472482.

Lahera G, Montes JM, Benito A, Valdivia M, Medina E, Mirapeix I, Sáiz-Ruiz J (2008). Theory of mind deficit in bipolar disorder: is it related to a previous history of psychotic symptoms? Psychiatry Research 161, 309-317.

Lahera G, Ruiz-Murugarren S, Iglesias P, Ruiz-Bennasar C, Herrería E, Montes JM, Fernández-Liria A (2012). Social cognition and global functioning in bipolar disorder. Journal of Nervous Mental Disease 200, 135-141.

Lee J, Altshuler L, Glahn DC, Miklowitz DJ, Ochsner K, Green MF (2013). Social and nonsocial cognition in bipolar disorder and schizophrenia: relative levels of impairment. American Journal of Psychiatry 170, 334-341.

Martino DJ, Strejilevich SA, Fassi G, Marengo E, Igoa A (2011). Theory of mind and facial emotion recognition in euthymic bipolar I and bipolar II disorders. Psychiatry Research 189, 379-384.

McKinnon MC, Cusi AM, Macqueen GM (2010). Impaired theory of mind performance in patients with recurrent bipolar disorder: moderating effect of cognitive load. Psychiatry Research 177, 261-262.

Moher D, Liberati A, Tetzlaff J, Altman DG, Group PRISMA (2009). Preferred reporting items for systematic reviews and meta-analyses: the PRISMA statement. British Medical Journal 339, b2535.

Montag C, Ehrlich A, Neuhaus K, Dziobek I, Heekeren HR, Heinz A, Gallinat J (2010). Theory of mind impairments in euthymic bipolar patients. Journal of Affective Disorders 123, 264-269.

Nuechterlein KH, Barch DM, Gold JM, Goldberg TE, Green MF, Heaton RK (2004). Identification of separable cognitive factors in schizophrenia. Schizophrenia Research 72, 29-39.

Olley AL, Malhi GS, Bachelor J, Cahill CM, Mitchell PB, Berk M (2005). Executive functioning and theory of mind in euthymic bipolar disorder. Bipolar Disorders 7 (Suppl. 5), $43-52$.

Ozel-Kizil E, Baskak B, Uran P, Cihan B, Zivrali E, Ates E, Cangoz B (2012). Recognition of faux pas dysfunction in patients with schizophrenia, bipolar disorder, their unaffected relatives and healthy controls. European Neuropsychopharmacology 22, S306.

Purcell AL, Phillips M, Gruber J (2013). In your eyes: does theory of mind predict impaired life functioning in bipolar disorder? Journal of Affective Disorders 151, 1113-1119.

Robinson L (2010). Neuropsychological performance, emotion processing and psychosocial function in bipolar disorder. PhD thesis. Newcastle University (https://theses. ncl.ac.uk/dspace/handle/10443/1069).

Rosenthal R, Rosnow R (1991). Essentials of Behavioral Research: Methods and Data Analysis. McGraw-Hill: New York, NY.

Rossell SL, Van Rheenen TE (2013). Theory of mind performance using a story comprehension task in bipolar mania compared to schizophrenia and healthy controls. Cognitive Neuropsychiatry 18, 409-421.

Rowland JE, Hamilton MK, Vella N, Lino BJ, Mitchell PB, Green MJ (2013). Adaptive associations between social cognition and emotion regulation are absent in schizophrenia and bipolar disorder. Frontiers in Psychology 3, 607 .

Rubinsztein JS, Fletcher PC, Rogers RD, Ho LW, Aigbirhio FI, Paykel ES, Robbins TW, Sahakian BJ (2001). Decision-making in mania: a PET study. Brain 124, 25502563.

Sakarya A (2012). Association of ToM deficits with insight and other cognitive functions among remitted schizophrenia and bipolar disorder patients. Thesis. Ankara University.

Samamé C, Martino DJ, Strejilevich SA (2012). Social cognition in euthymic bipolar disorder: systematic review and meta-analytic approach. Acta Psychiatrica Scandinavica 125, 266-280.

Samamé C, Martino DJ, Strejilevich SA (2015). An individual task meta-analysis of social cognition in euthymic bipolar disorders. Journal of Affective Disorders $\mathbf{1 7 3}$ 146-153.

Sanchez-Moreno J, Martinez-Aran A, Tabarés-Seisdedos R, Torrent C, Vieta E, Ayuso-Mateos JL (2009). Functioning and disability in bipolar disorder: an extensive review. Psychotherapy and Psychosomatics 78, 285-297.

Sarfati Y, Hardy-Baylé MC (1999). How do people with schizophrenia explain the behaviour of others? A study of theory of mind and its relationship to thought and speech disorganization in schizophrenia. Psychological Medicine 29, 613-620.

Schurz M, Radua J, Aichhorn M, Richlan F, Perner J (2014). Fractionating theory of mind: a meta-analysis of functional brain imaging studies. Neuroscience and Biobehavioral Reviews 42, 9-34.

Senju A (2012). Spontaneous theory of mind and its absence in autism spectrum disorders. Neuroscientist 18, 108-113.

Sergi MJ, Rassovsky Y, Widmark C, Reist C, Erhart S, Braff DL, Marder SR, Green MF (2007). Social cognition in schizophrenia: relationships with neurocognition and negative symptoms. Schizophrenia Research 90, 316-324.

Shamay-Tsoory S, Harari H, Szepsenwol O, Levkovitz Y (2009). Neuropsychological evidence of impaired cognitive 
empathy in euthymic bipolar disorder. Journal of Neuropsychiatry Clinical Neuroscience 21, 59-67.

Simon M, Herold R, Hajnal AS, Varga E, Fekete S, Tenyi T (2013). Theory of mind deficit in bipolar patients with subsyndromal illness. European Neuropsychopharmacology 23, S295-S296.

Stone VE, Baron-Cohen S, Knight RT (1998). Frontal lobe contributions to theory of mind. Journal of Cognitive Neuroscience 10, 640-656.

Stroup DF, Berlin JA, Morton SC, Olkin I, Williamson GD, Rennie D, Moher D, Becker BJ, Sipe TA, Thacker SB (2000). Meta-analysis of observational studies in epidemiology: a proposal for reporting. Meta-analysis Of Observational Studies in Epidemiology (MOOSE) group. Journal of the American Medical Association 283, 2008-2012.

Thaler NS, Allen DN, Sutton GP, Vertinski M, Ringdahl EN (2013). Differential impairment of social cognition factors in bipolar disorder with and without psychotic features and schizophrenia. Journal of Psychiatric Research 47, 2004-2010.

Trotta A, Murray RM, MacCabe JH (2014). Do premorbid and post-onset cognitive functioning differ between schizophrenia and bipolar disorder? A systematic review and meta-analysis. Psychological Medicine 45, 381-394.
Van Rheenen TE, Rossell SL (2013). Picture sequencing task performance indicates theory of mind deficit in bipolar disorder. Journal of Affective Disorders 151, 1132-1134.

Ventura J, Ered A, Gretchen-Doorly D, Subotnik KL, Horan WP, Hellemann GS, Nuechterlein KH (2015). Theory of mind in the early course of schizophrenia: stability, symptom and neurocognitive correlates, and relationship with functioning. Psychological Medicine 45, 2031-2043.

Viechtbauer W (2010). Conducting meta-analyses in R with the metafor package. Journal of Statistical Softwares 36 , $1-48$.

Wallace BC, Dahabreh IJ, Trikalinos TA, Lau J, Trow P, Schmid CH (2012). Closing the gap between methodologists and end-users: $\mathrm{R}$ as a computational back-end. Journal of Statistical Softwares 49, 1-15.

Wiener D, Andrzejewska M, Bodnar A, Rybakowski J (2011). Disturbances of the theory of mind and empathy in schizophrenia and bipolar affective illness. Neuropsychiatria i Neuropsychologia 6, 85-92.

Wolf F, Brüne M, Assion HJ (2010). Theory of mind and neurocognitive functioning in patients with bipolar disorder. Bipolar Disorders 12, 657-666. 